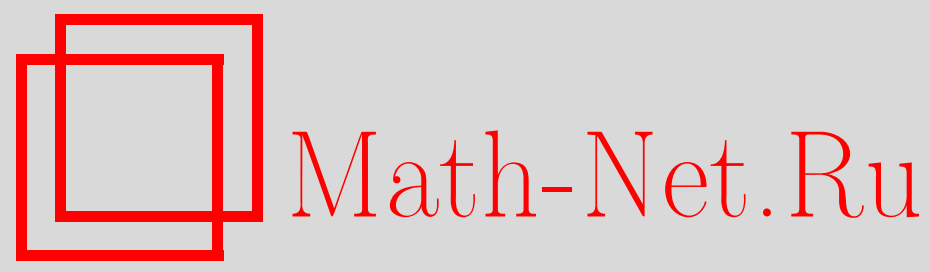

С. П. Горшков, А. В. Тарасов, О максимальных группах инвариантных преобразований мультиаффинных, биюнктивных, слабо положительных и слабо отрицательных булевых функций, Дискрет. матем., 2009, том 21, выпуск 2, 94-101

DOI: https://doi.org/10.4213/dm1049

Использование Общероссийского математического портала Math-Net.Ru подразумевает, что вы прочитали и согласны с пользовательским соглашением http://www . mathnet.ru/rus/agreement

Параметры загрузки:

IP : 54.198 .55 .26

26 апреля 2023 г., $15: 28: 59$ 


\title{
О максимальных группах инвариантных преобразований мультиаффинных, биюнктивных, слабо положительных и слабо отрицательных булевых функций
}

\author{
() 2009 г. С. П. Горшков, А. В. Тарасов
}

В работе изучаются некоторые свойства мультиаффинных, биюнктивных, слабо положительных и слабо отрицательных булевых функций. Доказаны следующие результаты: для любого целого $k \geqslant 1$ максимальной группой преобразований области определения функций от $k$ переменных, относительно которых множество мультиаффинных булевых функций инвариантно, является полная аффинная группа $A G L(k, 2)$; для биюнктивных функций при $k \geqslant 3-$ группа преобразований, каждое из которых есть комбинация перестановки и инверсии переменных функций; а для слабо положительных (слабо отрицательных) функций при $k \geqslant 2-$ группа преобразований, каждое из которых есть перестановка переменных функций.

\section{1. Введение}

Булева функция $f\left(x_{1}, \ldots, x_{k}\right)$ называется

(1) мультиаффинной, если существует представление $f$ в виде

$$
f\left(x_{1}, \ldots, x_{k}\right) \equiv \bigwedge_{i=1}^{t}\left(a_{i 1} x_{1} \oplus \ldots \oplus a_{i k} x_{k} \oplus a_{i 0}\right),
$$

где $a_{i j} \in\{0,1\}, i=1, \ldots, t, j=0,1, \ldots, k, 1 \leqslant t \leqslant k$;

(2) биюнктивной, если $f \equiv 1$ или существует представление функции $f$ в виде конъюнктивной нормальной формы (КНФ)

$$
f\left(x_{1}, \ldots, x_{k}\right) \equiv\left(\bigwedge_{i=1}^{t} x_{s_{i}}^{\alpha_{i}}\right) \wedge\left(\bigwedge_{j=1}^{q}\left(x_{r_{j 1}}^{\beta_{j 1}} \vee x_{r_{j 2}}^{\beta_{j 2}}\right)\right)
$$

где $\alpha_{i}, \beta_{j 1}, \beta_{j 2} \in\{0,1\}, s_{i}, r_{j 1}, r_{j 2} \in\{1, \ldots, k\}, i=1, \ldots, t, j=1, \ldots, q$;

(3) слабо положительной, если $f \equiv 1$ или существует представление $f$ в виде КНФ

$$
f\left(x_{1}, \ldots, x_{k}\right) \equiv \bigwedge_{i=1}^{t}\left(x_{r_{i 1}}^{a_{i}} \vee x_{r_{i 2}} \vee \ldots \vee x_{r_{i k_{i}}}\right),
$$


где $\alpha_{i} \in\{0,1\}, k_{i} \leqslant k, r_{i j} \in\{1, \ldots, k\}, i=1, \ldots, t, j=1, \ldots, k_{i} ;$

(4) слабо отрицательной, если $f \equiv 1$ или существует представление $f$ в виде КНФ

$$
f\left(x_{1}, \ldots, x_{k}\right) \equiv \bigwedge_{i=1}^{t}\left(x_{r_{i 1}}^{a_{i}} \vee \bar{x}_{r_{i 2}} \vee \ldots \vee \bar{x}_{r_{i k_{i}}}\right),
$$

где $\alpha_{i} \in\{0,1\}, k_{i} \leqslant k, r_{i j} \in\{1, \ldots, k\}, i=1, \ldots, t, j=1, \ldots, k_{i}$.

Множества всех мультиаффинных, биюнктивных, слабо положительных, слабо отрицательных функций обозначим, соответственно, $A, B i, W P, W N$. Функции из классов (1)-(4), зависящие от $k$ переменных, обозначим $A_{k}, B i_{k}, W P_{k}, W N_{k}$.

$\mathrm{B}[1,2]$ показано, что мультиаффинные, биюнктивные, слабо положительные и слабо отрицательные функции порождают полиномиально решаемые классы систем булевых уравнений без ограничений на выбор неизвестных (такие классы также называют классами систем с ограничениями на выбор функций и без ограничений на выбор неизвестных). В работах [1-5] исследованы некоторые свойства указанных функций. В данной статье изучаются максимальные группы инвариантных преобразований мультиаффинных, биюнктивных, слабо положительных и слабо отрицательных булевых функций.

Пусть $\mathbf{N}-$ множество натуральных чисел; $\mathbf{N}_{0}=\mathbf{N} \cup\{0\} ; B_{k}-$ множество двоичных $k$-мерных векторов; $B_{k}^{(i)}=\left\{\alpha \in B_{k}|| \alpha \|=i\right\}$, где $i \in\{0,1, \ldots, k\}$ (слой векторов веса $i) ; F_{k}-$ множество булевых функций от $k \in \mathbf{N}_{0}$ переменных; $\operatorname{Sym}(n)-$ симметрическая группа степени $n ; S_{2^{k}}$ - симметрическая группа перестановок на множестве $B_{k}$, изоморфная $\operatorname{Sym}\left(2^{k}\right) ; \widetilde{S}_{k}-$ симметрическая группа перестановок координат векторов из $B_{k}$, подгруппа $S_{2^{k}}$, изоморфная $\operatorname{Sym}(k) ; \Sigma_{k}-$ группа сдвигов, она является подгруппой $\operatorname{Sym}\left(2^{k}\right)$, состоит из преобразований $\tau_{\beta}(x)=x \oplus \beta$, где $x, \beta \in B_{k} ; \oplus-$ покоординатное сложение по модулю $2 ; Q_{k}$ - группа Джевонса, являющаяся полупрямым произведением групп $\widetilde{S}_{k}$ и $\Sigma_{k}$, она состоит из преобразований вида $s_{\pi \beta}(x)=\tau_{\beta}(\pi(x))$, где $\tau_{\beta} \in \Sigma_{k}$, $\pi \in \widetilde{S}_{k} ; A G L(k, 2)$ - полная аффинная группа преобразований пространства $B_{k}$, где каждый элемент $T \in A G L(k, 2)$ имеет вид $T=\tau_{\beta}(l(x)), \tau_{\beta} \in \Sigma_{k}$, a $l$ - невырожденное линейное преобразование пространства $B_{k}$.

Двоичный вектор $\left(\alpha_{1}, \ldots, \alpha_{k}\right)$ назовем выполняющим вектором функции $f\left(x_{1}, \ldots, x_{k}\right)$, если $f\left(\alpha_{1}, \ldots, \alpha_{k}\right)=1$. Множество всех выполняющих векторов функции $f$ обозначим $E_{f}$.

Для произвольной булевой функции $f \in F_{k}$ с множеством выполняющих векторов $E_{f}$ и $s \in S_{2^{k}}$ пусть $f^{s}-$ булева функция с множеством выполняющих векторов $s\left(E_{f}\right)$.

Если $\alpha=\left(\alpha_{1}, \ldots, \alpha_{k}\right), \beta=\left(\beta_{1}, \ldots, \beta_{k}\right)-$ двоичные векторы, $f\left(x_{1}, \ldots, x_{k}\right)-$ булева функция, то полагаем

$$
\begin{aligned}
& \alpha \vee \beta=\left(\alpha_{1} \vee \beta_{1}, \ldots, \alpha_{k} \vee \beta_{k}\right), \\
& \alpha \wedge \beta=\left(\alpha_{1} \wedge \beta_{1}, \ldots, \alpha_{k} \wedge \beta_{k}\right) .
\end{aligned}
$$

Рассмотрим следующие свойства (см. [1, 3]).

Свойство 1 (критерий биюнктивности). Функция $f\left(x_{1}, \ldots, x_{k}\right)$ является биюнктивной, если и только если для любых трех выполняющих векторов $\{\alpha, \beta, \gamma\} \subseteq E_{f}$ этой функции справедливо включение $(\alpha \wedge \beta) \vee(\alpha \wedge \gamma) \vee(\beta \wedge \gamma) \in E_{f}$.

Свойство 2 (критерий слабой положительности). Функция $f\left(x_{1}, \ldots, x_{k}\right)$ является слабо положительной, если и только если для любых двух выполняющих векторов $\{\alpha, \beta\} \subseteq E_{f}$ этой функции справедливо включение $\alpha \vee \beta \in E_{f}$. 
Свойство 3 (критерий слабой отрицательности). Функция $f\left(x_{1}, \ldots, x_{k}\right)$ является слабо отрицательной, если и только если для любых двух выполняющих векторов $\{\alpha, \beta\} \subseteq E_{f}$ этой функции справедливо включение $\alpha \wedge \beta \in E_{f}$.

Пусть $M_{k} \subseteq F_{k}, k \in \mathbf{N}$. Максимальную группу преобразований области определения булевых функций от $k$ переменных, относительно которых множество функций $M_{k}$ инвариантно, обозначим символом $G_{M_{k}}$, то есть

$$
G_{M_{k}}=\left\{s \in S_{2^{k}}: f^{s} \in M_{k} \text { для всех } f \in M_{k}\right\} .
$$

Булеву функцию $f \in F_{k}$ называем равновероятной, если ее вес равен $2^{k-1}$.

Рассматривая подстановку $s \in S_{2^{k}}$ как вектор-функцию из $B_{k}$ в $B_{k}$, обозначим ее координатные функции через $s_{1}, \ldots, s_{k}$, то есть $s=\left(s_{1}, \ldots, s_{k}\right)$. Поскольку $s-$ подстановка на $B_{k}$, все координатные функции $s_{1}, \ldots, s_{k}$ являются равновероятными.

\section{2. Максимальная группа инвариантных преобразований множества мультиаффинных функций}

Лемма 1. Для произвольной функциии $f \in F_{k}$ и подстановки $s \in S_{2}$ верно равенство

$$
f^{s}\left(x_{1}, \ldots, x_{k}\right)=f\left(s^{-1}\left(x_{1}, \ldots, x_{k}\right)\right) .
$$

Доказательство. В самом деле,

$$
f^{s}(x)=1 \Longleftrightarrow x \in s\left(E_{f}\right)
$$

С другой стороны,

$$
f\left(s^{-1}(x)\right)=1 \Longleftrightarrow s^{-1}(x) \in E_{f} \Longleftrightarrow x \in s\left(E_{f}\right) .
$$

Лемма доказана.

Лемма 2. Пусть $M_{k} \subseteq F_{k}-$ класс булевых функций от $k$ переменных, содержащий функции $f_{i}\left(x_{1}, \ldots, x_{k}\right)=x_{i}, g_{i}\left(x_{1}, \ldots, x_{k}\right)=\bar{x}_{i}, i \in\{1, \ldots, k\}, s \in G_{M_{k}}$. Тогда координатные функции $s_{1}, \ldots, s_{k}$ подстановки s удовлетворяют следуюшим условиям:

(1) $s_{1}, \ldots, s_{k} \in M_{k}$.

(2) $\bar{s}_{1}, \ldots, \bar{s}_{k} \in M_{k}$.

Доказательство. Для $i \in\{1, \ldots, k\}$ рассмотрим функции $f_{i}, g_{i}$. Так как $s \in G_{M_{k}}$, справедливо включение $s^{-1} \in G_{M_{k}}$. В этом случае функции $f_{i}^{s^{-1}}, g_{i}^{s^{-1}}=\bar{f}_{i}^{s^{-1}}$ также лежат в $M_{k}$. По лемме $1, f_{i}^{s^{-1}}=s_{i}, g_{i}^{s^{-1}}=\bar{s}_{i}$, откуда следует утверждение леммы.

Теорема 1. Справедливо соотношение $G_{A_{k}}=A G L(k, 2)$.

Доказательство. Включение $A G L(k, 2)<G_{A_{k}}$ очевидно. Пусть $L_{k}-$ класс аффинных функций от $k$ переменных и $s=\left(s_{1}, \ldots, s_{k}\right) \in G_{A_{k}}$. Тогда, по лемме $2, s_{1}, \ldots, s_{k} \in A_{k}$ и $s_{1}, \ldots, s_{k}$ равновероятны. Следовательно, $s_{i} \in L_{k}, i=1,2, \ldots, k$. Таким образом, $s-$ подстановка, все координатные функции которой являются аффинными. Следовательно, $s \in A G L(k, 2)$. Теорема доказана. 
Замечание 1. Приведем другое доказательство теоремы 1, которое принадлежит А. В. Черемушкину. Справедливость включения $A G L(k, 2)<G_{A_{k}}$ очевидна. Если $k \leqslant 2$, то $G_{A_{k}}=A G L(k, 2)=S_{2^{k}}$. При $k \geqslant 3$ группа $A G L(k, 2)$ является максимальной подгруппой $S_{2^{k}}[6]$. Если бы $G_{A_{k}}=S_{2^{k}}$, то множество мультиаффинных функций от $k \geqslant 3$ переменных содержало все равновероятные функции, а это не так. Поэтому $G_{A_{k}}=A G L(k, 2)$.

\section{3. Максимальная группа инвариантных преобразований множества биюнктивных функций}

При $k \leqslant 2$ справедливо равенство $G_{B i_{k}}=S_{2^{k}}$, поскольку все булевы функции от $k \leqslant 2$ переменных являются биюнктивными.

Функция $f \in B i_{k}$ называется дважды биюнктивной, если $\bar{f} \in B i_{k}$. В [2] рассмотрены дважды биюнктивные функции и показано, что они существенно зависят не более, чем от 4 переменных. При этом равновероятные дважды биюнктивные функции исчерпываются следующими классами геометрической эквивалентности (класс эквивалентности булевых функций относительно перестановки переменных, навешивания отрицания на переменные и инвертирования всей функции): $\left[x_{1}\right],\left[x_{1} \oplus x_{2}\right],\left[\left(x_{1} \vee x_{2}\right)\left(x_{1} \vee x_{3}\right)\left(x_{2} \vee x_{3}\right)\right]$, $\left[\left(x_{1} \vee x_{2}\right)\left(\bar{x}_{1} \vee x_{3}\right)\right],\left[\left(x_{1} \vee x_{2}\right)\left(x_{1} \vee x_{3}\right)\left(x_{2} \vee x_{4}\right)\right]$.

Множество дважды биюнктивных функций от $k$ переменных обозначим $2 B i_{k}$, а множество равновероятных функций из $2 B i_{k}$ обозначим $2 B i_{k}^{\prime}$.

Лемма 3. Если $s \in G_{B i_{k}}$, то функции $s_{1}, \ldots, s_{k}$ являются равновероятными дважды биюнктивными функциями.

Доказательство. Легко видеть, что если $s \in G_{B i_{k}}$, то $s \in G_{2 B i_{k}^{\prime}}$, поэтому $G_{B i_{k}}<G_{2 B i_{k}^{\prime}}$. Тогда, согласно лемме 2 , для $s=\left(s_{1}, \ldots, s_{k}\right)$ справедливы включения $s_{1}, \ldots, s_{k} \in 2 B i_{k}^{\prime}$. Лемма доказана.

Ясно, что $Q_{k}<G_{B i_{k}}$, поэтому для $s \in G_{B i_{k}}$ включение $s \in Q_{k}$ выполняется тогда и только тогда, когда $s^{-1} \in Q_{k}$. При этом, если все координатные функции подстановки $s$ существенно зависят ровно от одной переменной, то эта подстановка лежит в $Q_{k}$. Биективность отображения $s$ влечет также следующий факт: для любых $1 \leqslant i_{1}<\ldots<i_{l} \leqslant k$ вектор-функция $s_{i_{1} \ldots i_{l}}\left(x_{1}, \ldots, x_{k}\right)=\left(s_{i_{1}}\left(x_{1}, \ldots, x_{k}\right), \ldots, s_{i_{l}}\left(x_{1}, \ldots, x_{k}\right)\right)$ существенно зависит не менее чем от $l$ переменных. Пусть $I(f)$ - множество существенных переменных функции $f$.

Лемма 4. Пусть $k \geqslant 3, s \in G_{B i_{k}}$, и все функциии $s_{1}, \ldots, s_{k}$ сущуественно зависят не более чем от двух переменных. Тогда функции $s_{1}, \ldots, s_{k}$ сущеественно зависят от одной переменной и $s \in Q_{k}$.

Доказательство. Поскольку $s \in G_{B i_{k}}$, справедливо включение $s^{-1} \in G_{B i_{k}}$. Функции $s_{1}, \ldots, s_{k}$ существенно зависят не более чем от двух переменных и в силу леммы 3 являются равновероятными дважды биюнктивными функциями, поэтому они являются аффинными функциями, существенно зависящими не более чем от двух переменных. Предположим, что среди $k$ указанных функций $l$ функций существенно зависят от одной переменной, а $k-l-$ от двух переменных. Без ограничения общности будем считать, что

$$
\begin{gathered}
s_{1}=x_{i_{1}} \oplus a_{1}, \ldots, s_{l}=x_{i_{l}} \oplus a_{l}, \\
s_{l+1}=x_{j_{1,1}} \oplus x_{j_{1,2}} \oplus a_{l+1}, \ldots, s_{k}=x_{j_{k-l, 1}} \oplus x_{j_{k-l, 2}} \oplus a_{k} .
\end{gathered}
$$


Поскольку $s-$ подстановка, все координатные функции которой являются аффинными функциями, соответствующая матрица должна быть невырожденной.

Рассмотрим функции $s_{m}, s_{n}, 1 \leqslant m<n \leqslant k$. Пусть $\left|I\left(s_{m}\right) \cup I\left(s_{n}\right)\right| \geqslant 3$ для некоторых $m, n$. Если $I\left(s_{m}\right) \cap I\left(s_{n}\right)=\varnothing$, то, рассмотрев функции $f\left(x_{1}, \ldots, x_{k}\right)=x_{m} \oplus x_{n} \in B i_{k}$, $f^{s^{-1}}=s_{m} \oplus s_{n}$, легко показать, что $f^{s^{-1}} \notin B i_{k}$. В самом деле, в указанном случае либо $f^{s^{-1}}=x_{p_{1}} \oplus x_{p_{2}} \oplus x_{p_{3}} \oplus b$, либо $f^{s^{-1}}=x_{p_{1}} \oplus x_{p_{2}} \oplus x_{p_{3}} \oplus x_{p_{4}} \oplus b$ для подходящих $1 \leqslant p_{1}, \ldots, p_{4} \leqslant k, b \in\{0,1\}$. Указанные функции не являются биюнктивными и лемма верна.

Если же $\left|I\left(s_{m}\right)\right|=\left|I\left(s_{n}\right)\right|=2$ и $I\left(s_{m}\right) \cap I\left(s_{n}\right) \neq \varnothing$, то $\left|I\left(s_{m}\right) \cap I\left(s_{n}\right)\right|=1$. Рассмотрим функцию $f=x_{m} \vee x_{n}$. Тогда $f^{s^{-1}}=\left(x_{p_{1}} \oplus x_{p_{2}} \oplus b_{1}\right) \vee\left(x_{p_{2}} \oplus x_{p_{3}} \oplus b_{2}\right)$ для некоторых $1 \leqslant p_{1}, p_{2}, p_{3} \leqslant k, b_{1}, b_{2} \in\{0,1\}$. Без ограничения общности будем считать, что $p_{1}=1, p_{2}=2, p_{3}=3$. Тогда $f^{s^{-1}}=\left(x_{1} \oplus x_{2} \oplus b_{1}\right) \vee\left(x_{2} \oplus x_{3} \oplus b_{2}\right)$. Эта функция биюнктивна тогда и только тогда, когда биюнктивна функция $g=f^{s^{-1}}\left(x_{1} \oplus b_{1}, x_{2}, x_{3} \oplus b_{2}\right)=\left(x_{1} \oplus x_{2}\right) \vee\left(x_{2} \oplus x_{3}\right)$. Рассмотрим выполняющие векторы $a=(0010 \ldots 0), b=(0100 \ldots 0), c=(1000 \ldots 0)$ функции $g$. Вектор $d=a b \vee a c \vee b c=$ $(000 \ldots 0) \notin E_{g}$. Следовательно, функция $f^{s^{-1}}$ также не является биюнктивной. Таким образом, случай $\left|I\left(s_{m}\right) \cap I\left(s_{n}\right)\right| \geqslant 3$ невозможен.

Остался случай, когда для всех $m, n, 1 \leqslant m<n \leqslant k$,

$$
\left|I\left(s_{m}\right) \cup I\left(s_{n}\right)\right|=2, \quad\left|I\left(s_{m}\right)\right|+\left|I\left(s_{n}\right)\right| \leqslant 3 .
$$

Поэтому для всех $1 \leqslant m<n \leqslant k$ выполняется одно из условий $\left|I\left(s_{m}\right)\right|=\left|I\left(s_{n}\right)\right|=1$ или $\left|I\left(s_{m}\right)\right|=1,\left|I\left(s_{n}\right)\right|=2, I\left(s_{m}\right) \subset I\left(s_{n}\right)$.

Пусть $\left|I\left(s_{m}\right)\right|=1,\left|I\left(s_{n}\right)\right|=2, I\left(s_{m}\right) \subset I\left(s_{n}\right)$ для некоторых $m, n$. Без ограничения общности считаем, что $m=1, n=2, I\left(s_{1}\right)=\left\{x_{1}\right\}, I\left(s_{2}\right)=\left\{x_{1}, x_{2}\right\}$. Тогда в силу того, что $k \geqslant 3$, существует функция $s_{j}$, существенно зависящая от переменной $x_{i} \notin\left\{x_{1}, x_{2}\right\}$. В этом случае $\left|I\left(s_{2}\right)\right| \cup I\left(s_{j}\right) \mid \geqslant 3$, что противоречит условию (5).

Если же $\left|I\left(s_{m}\right)\right|=\left|I\left(s_{n}\right)\right|=1$ для всех $1 \leqslant m, n \leqslant k$, то лемма верна, так как в этом случае $s \in Q_{k}$, а значит, $s^{-1} \in Q_{k}$. Лемма доказана.

Лемма 5. Для любых различных векторов $a, b, c, d \in B_{k}, k \geqslant 3$, таких, что $d \neq a b \vee a c \vee b c$, найдется такая функиия $f \in B i_{k}$, что $f(a)=f(b)=f(c)=1$, но $f(d)=0$.

Доказательство. Рассмотрим функцию $f \in B i_{k}$ такую, что множество $E_{f}$ содержит все различные векторы из векторов $a, b, c, a b \vee a c \vee b c$. Тогда $3 \leqslant\|f\| \leqslant 4, d \notin E_{f}$. Следовательно, $f(a)=f(b)=f(c)=1$ и $f(d)=0$. Лемма доказана.

Лемма 6. Пусть в подстановке $s \in G_{B i_{k}}$ координатная функиия $s_{i}, i \in\{1, \ldots, k\}, c y$ щзественно зависит от трех переменных. Тогда найдутся такие три вектора $a, b, c \in B_{k}$, что $a b \vee a c \vee b c \notin\{a, b, c\}, s(a) s(b) \vee s(a) s(c) \vee s(b) s(c) \neq s(a b \vee a c \vee b c)$.

Доказательство. Функция $s_{i}$ является дважды биюнктивной равновероятной, существенно зависящей от 3 переменных, поэтому $s_{i} \in\left[\left(x_{1} \vee x_{2}\right)\left(x_{2} \vee x_{3}\right)\left(x_{1} \vee x_{3}\right)\right]$ или $s_{i} \in\left[\left(x_{1} \vee x_{2}\right)\left(\bar{x}_{1} \vee x_{3}\right)\right]$. Без ограничения общности будем считать, что $i=1$ и либо $s_{1}=\left(x_{1} \vee x_{2}\right)\left(x_{2} \vee x_{3}\right)\left(x_{1} \vee x_{3}\right)$, либо $s_{1}=\left(x_{1} \vee x_{2}\right)\left(\bar{x}_{1} \vee x_{3}\right)$. Следующие рассуждения верны в обоих случаях. Положим $a=(1010 \ldots 0), b=(0110 \ldots 0), c=(0000 \ldots 0)$. Тогда $a b \vee a c \vee b c=(0010 \ldots 0) \notin\{a, b, c\}$. Поэтому $(s(a b \vee a c \vee b c))_{1}=s_{1}(a b \vee a c \vee b c)=0$. C другой стороны, выполнены равенства $(s(a))_{1}=1,(s(b))_{1}=1,(s(c))_{1}=0$, откуда следует, что $(s(a) s(b) \vee s(a) s(c) \vee s(b) s(c))_{1}=1$. Следовательно, $s(a) s(b) \vee s(a) s(c) \vee s(b) s(c) \neq$ $s(a b \vee a c \vee b c)$. Лемма доказана. 
Лемма 7. Пусть в подстановке $s$ координатная функция $s_{i}, i \in\{1, \ldots, k\}$, сущчественно зависит от четырех переменных. Тогда найдутся такие три вектора $a, b, c \in B_{k}$, что $a b \vee a c \vee b c \notin\{a, b, c\}, s(a) s(b) \vee s(a) s(c) \vee s(b) s(c) \neq s(a b \vee a c \vee b c)$.

Доказательство. Доказательство данной леммы аналогично доказательству леммы 6 . Поскольку $s_{i}$ - дважды биюнктивная равновероятная функция, существенно зависящая от 4 переменных, справедливо включение $s_{i} \in\left[\left(x_{1} \vee x_{2}\right)\left(x_{2} \vee x_{3}\right)\left(x_{3} \vee x_{4}\right)\right]$. Без ограничения общности будем считать, что $i=1, s_{1}=\left(x_{1} \vee x_{2}\right)\left(x_{2} \vee x_{3}\right)\left(x_{3} \vee x_{4}\right)$. Положим $a=(01010 \ldots 0), b=(10110 \ldots 0), c=(00000 \ldots 0)$. Тогда $a b \vee a c \vee b c=$ $(00010 \ldots 0)$. Поэтому $(s(a b \vee a c \vee b c))_{1}=0$. С другой стороны, $(s(a))_{1}=1,(s(b))_{1}=1$, $(s(c))_{1}=0$, откуда следует, что $(s(a) s(b) \vee s(a) s(c) \vee s(b) s(c))_{1}=1$. Следовательно, $s(a) s(b) \vee s(a) s(c) \vee s(b) s(c) \neq s(a b \vee a c \vee b c)$. Лемма доказана.

После рассмотрения предварительных лемм, можно перейти к доказательству основной теоремы.

Теорема 2. При $k \geqslant 3$ выполнено равенство $G_{B i_{k}}=Q_{k}$.

Доказательство. Включение $Q_{k} \subseteq G_{B i_{k}}$ очевидно. Пусть теперь $s \in G_{B i_{k}}$, но $s \notin Q_{k}$. Если $s=\left(s_{1}, \ldots, s_{k}\right)$ - покоординатная запись подстановки $s$, то среди функций $s_{1}, \ldots, s_{k}$ должны найтись функции, существенно зависящие более чем от одной переменной. Кроме того, по лемме 3 функции $s_{1}, \ldots, s_{k}$ являются равновероятными дважды биюнктивными функциями. Следовательно, каждая из функций $s_{1}, \ldots, s_{k}$ существенно зависит не более чем от 4 переменных.

Если все функции $s_{1}, \ldots, s_{k}$ существенно зависят не более чем от двух переменных, то $s^{-1} \in Q_{k}$ по лемме 4, следовательно, $s \in Q_{k}$, что противоречит исходному предположению. Пусть среди функций $s_{1}, \ldots, s_{k}$ есть функции, существенно зависящие от 3 или 4 переменных. Для этого случая воспользуемся леммами 6 и 7. Пусть $a, b, c \in B_{k}-$ векторы такие, что $s(a) s(b) \vee s(a) s(c) \vee s(b) s(c) \neq s(a b \vee a c \vee b c)$. Пусть $g-$ биюнктивная функция такая, что $E_{g}=\{a, b, c, a b \vee a c \vee b c\}$. Тогда, по лемме $1, E_{g^{-1}}=s\left(E_{g}\right)=$ $\{s(a), s(b), s(c), s(a b \vee a c \vee b c)\}$. При этом $s(a) s(b) \vee s(a) s(c) \vee s(b) s(c) \neq s(a b \vee a c \vee b c)$. Если $s(a) s(b) \vee s(a) s(c) \vee s(b) s(c) \notin\{s(a), s(b), s(c)\}$, то функция $g^{s^{-1}}$ не биюнктивна. Следовательно, $s(a) s(b) \vee s(a) s(c) \vee s(b) s(c) \in\{s(a), s(b), s(c)\}$. Но тогда функция $h: E_{h}=\{s(a), s(b), s(c)\}$ биюнктивна. Следовательно, биюнктивной будет и функция $h^{s}: E_{h^{s}}=\{a, b, c\}$, откуда следует включение $a b \vee a c \vee b c \in\{a, b, c\}$, противоречащее условиям использованных лемм 6 и 7. Полученные противоречия доказывают теорему.

\section{4. Максимальная группа инвариантных преобразований множества слабо положительных (слабо отрицательных) функций}

При $k=1$ справедливо равенство $G_{W P_{1}}=S_{2^{1}}$, поскольку все булевы функции от одного переменного являются слабо положительными.

Всякой подстановке $r=\left(\begin{array}{ccc}1 & \ldots & k \\ r_{1} & \ldots & r_{k}\end{array}\right) \in \operatorname{Sym}(k)$ сопоставим подстановку $s_{r} \in S_{2_{k}}$ по следующему правилу: для любого вектора $\alpha=\left(\alpha_{1}, \ldots, \alpha_{k}\right) \in B_{k}$

$$
s_{r}(\alpha)=\left(\alpha_{r_{1}}, \ldots, \alpha_{r_{k}}\right)
$$


При этом

$$
\widetilde{S}_{k}=\left\{s_{r} \in S_{2^{k}} \mid r \in \operatorname{Sym}(k)\right\} .
$$

Теорема 3. При $k \geqslant 2$ выполняется равенство $G_{W P_{k}}=\widetilde{S}_{k}$.

Доказательство. Для любой подстановки $s \in S_{2^{k}}$ и любой функции $f \in F_{k}$ выполняется равенство $\|f\|=\left\|f^{s}\right\|$, где $\|f\|-$ вес функции $f$.

Включение $G_{W P_{k}} \supseteq \widetilde{S}_{k}$ очевидно. Докажем обратное включение $G_{W P_{k}} \subseteq \widetilde{S}_{k}$.

Пусть $f \in W P_{k},\|f\|=2^{k}-1$. Тогда функция $f$ имеет вид

$$
f \equiv x_{1} \vee \ldots x_{j-1} \vee x_{j}^{\beta} \vee x_{j+1} \vee \ldots \vee x_{k},
$$

где $j \in\{1, \ldots, k\}, \beta \in\{0,1\}$. Множество функций вида (7) обозначим $F^{\prime}$. Поскольку при любом преобразовании $s \in G_{W P_{k}}$ сохраняется вес функции, $f^{s} \in F^{\prime}$ для всякой функции $f \in F^{\prime}$, то есть справедливо следующее свойство: для любой подстановки $s \in G_{W P_{k}}$, для любого элемента $\alpha \in B_{k}^{(0)} \cup B_{k}^{(1)}$ выполняется соотношение $s(\alpha) \in B_{k}^{(0)} \cup B_{k}^{(1)}$.

Покажем, что для любой подстановки $s \in G_{W P_{k}}$ верно равенство $s\left(0_{k}\right)=0_{k}$, где $0_{k}=(0, \ldots, 0) \in B_{k}$. Предположим противное, то есть предположим, что найдется $s \in G_{W P_{k}}$ такая, что $s\left(0_{k}\right) \in B_{k}^{(1)}$. Для простоты записи положим $s\left(0_{k}\right)=(1,0, \ldots, 0)$. Введем обозначение $\alpha^{(1)}=(1,0, \ldots, 0)$.

Из критерия слабой положительности следует, что функция $f$ веса 2 такая, что $\|f\|=2, E_{f}=\left\{\alpha^{\prime}, \alpha^{\prime \prime}\right\}$, является слабо положительной, если и только если

$$
\alpha^{\prime}<\alpha^{\prime \prime} \quad \text { или } \alpha^{\prime \prime}<\alpha^{\prime} \text {. }
$$

Ясно, что функция $f_{1}$ веса 2 , для которой $E_{f_{1}}=\left\{0_{k}, \alpha^{(1)}\right\}$, является слабо положительной. Рассмотрим функцию $f_{1}^{s}$, для которой $E_{f_{1}^{s}}=\left\{\alpha^{(1)}, s\left(\alpha^{(1)}\right)\right\}$. Если $s\left(\alpha^{(1)}\right) \neq 0_{k}$, то $f_{1}^{s} \notin W P$. Далее считаем, что $s\left(\alpha^{(1)}\right)=0_{k}$.

Так как $k \geqslant 2$, среди функций $W P_{k}$ имеется слабо положительная функция веса 2 с множеством выполняющих векторов $\{(0, \ldots, 0),(0,1,0, \ldots, 0)\}$. Эту функцию обозначим $f_{2}$. Из приведенных выше свойств следует, что $s\left(0_{k}\right)=\alpha^{(1)} \in B_{k}^{(1)}, s(010 \ldots 0) \in B_{k}^{(1)}$, поэтому $f_{2}^{s} \notin W P$. Пришли к противоречию с предположением, что $s\left(0_{k}\right) \in B_{k}^{(1)}$. Поэтому $s\left(0_{k}\right)=0_{k}$ для любого $s \in G_{W P_{k}}$.

По доказанному выше для любого $s \in G_{W P_{k}}$ множество $s\left(B_{k}^{(1)}\right)$ есть перестановка элементов $B_{k}^{(1)}$. Для всякого преобразования $s \in G_{W P_{k}}$ определим подстановку $r \in \operatorname{Sym}(k)$ по правилу $r=\left(\begin{array}{ccc}1 & \ldots & k \\ r_{1} & \ldots & r_{k}\end{array}\right) \in \operatorname{Sym}(k)$, где $\left.r_{i}, i \in\{1, \ldots, k\}\right)$, равно номеру единицы в векторе $s(0, \ldots, 0,1, \ldots, 0)$. Докажем, что

$$
s=s_{i}
$$

(см. (6)). Предположим противное, то есть предположим, что $s \neq s_{i}$. Выберем вектор $\alpha^{(2)} \in B_{k}$ с минимальным весом такой, что

$$
s\left(\alpha^{(2)}\right) \neq s_{r}\left(\alpha^{(2)}\right) .
$$

Ясно, что $\left\|\alpha^{(2)}\right\| \geqslant 2$. Если имеется несколько векторов с весом $\left\|\alpha^{(2)}\right\|$, для которых справедливо (10), то выберем любой их них. 
Пусть $\left\|\alpha^{(2)}\right\|=t$. Рассмотрим слабо положительную функцию $f_{3} \in W P_{k}$ веса $\left\|f_{3}\right\|=2^{t}$, множество $E_{f_{3}}$ выполняющих векторов которой равно

$$
E_{f_{3}}=\left\{\alpha \in B_{k} \mid \alpha \leqslant \alpha^{(2)}\right\}
$$

Для функции $f_{3}^{s}$ выполняются соотношения $s(\alpha)=s_{r}(\alpha) \in E_{f_{3}^{s}}$, если $\alpha<\alpha^{(2)}$, и $\left\|f_{3}^{s}\right\|=2^{t}$. Поскольку $f_{3} \in W P_{k}$, должны выполняться равенства

$$
\left(\bigvee_{\alpha<\alpha^{(2)}} s(\alpha)\right)=\left(\bigvee_{\alpha<\alpha^{(2)}} s_{r}(\alpha)\right)=s_{r}\left(\alpha^{(2)}\right) \in E_{f_{3}^{s}},
$$

ввиду (10) получаем, что $\left\|f_{3}^{s}\right\| \geqslant 2^{t}+1$. Пришли к противоречию с тем, что $\left\|f_{3}^{s}\right\|=2^{t}$, поэтому верны соотношения (9) и (7). Следовательно $G_{W P_{k}}=\widetilde{S}_{k}$. Теорема доказана.

Введем обозначение

$$
G_{W N_{k}}=\left\{s \in S_{2^{k}}: \forall f \in W N_{k} f^{s} \in W N_{k}\right\} .
$$

Теорема 4. При $k \geqslant 2$ выполняется равенство $G_{W P_{k}}=\widetilde{S}_{k}$.

Для доказательства теоремы достаточно воспользоваться теоремой 3 и тем, что функция $f\left(x_{1}, \ldots, x_{k}\right)$ является слабо отрицательной, если и только если $f\left(\bar{x}_{1}, \ldots, \bar{x}_{k}\right) \in W P$.

\section{Список литературы}

1. Schaefer T. J., Complexity of satisfiability problems. In: Proc. 10th STOC Conf. ACM Press, 1978, pp. 216-226.

2. Горшков С. П., Применение теории $N P$-полных задач для оценки сложности решения систем булевых уравнений. Обозрение прикладной и промышленной математики (1995) 2, №3, 325398.

3. Гизунов С. А., Носов В. А., О классификации всех булевых функций четырех переменных по классам Шефера. Обозрение прикладной и промышленной математики (1995) 2, №3, 440-467.

4. Тарасов А. В., О свойствах функций, представимых в виде 2-КНФ. Дискретная математика (2001) 13, №4, 99-115.

5. Горшков С. П., О сложности распознавания мультиаффинности, биюнктивности, слабой положительности и слабой отрицательности булевых функций. Обозрение прикладной и промышленной математики (1997) 4, №2, 216-237.

6. Погорелов Б. А., О максимальных подгруппах симметрических групп, заданных на проективных пространствах над конечным полем. Матем. заметки (1977) 16, №1, 91-100.

Статья поступила 20.03.2007. 